\title{
Maximal Coupling of Bianchi Type- II, VIII and IX Space-Time with HDE and $D M$ in $f(R, T)$ Gravity
}

\author{
S.R. Bhoyar, V.R. Chirde ${ }^{1}$, S.H. Shekh ${ }^{2}$ \\ Department of Mathematics, Phulsing Naik Mahavidyalaya, Pusad-445216,India \\ ${ }^{1}$ Department of Mathematics, Gopikabai Sitaramji Gawande College, Umarkhed-445206, India \\ ${ }^{2}$ Department of Mathematics, Iawaharlal Darda Institute of Engg.\& Technology Yavatmal-445001, India \\ *Corresponding Author: sbhoyar68@yahoo.com Tel.: +91-9422583592
}

Available online at: www.isroset.org

Received: 23/Mar/2018, Accepted: 19/Apr/2018, Online: 31/Dec/2018

\begin{abstract}
The present investigation represents the study the role of Non-Viscous and Viscous Holographic Dark Energy (HDE) and Dark Matter (DM) for a homogeneous anisotropic Bianchi type-II, type-VIII and type-IX cosmological models within the frame work of a maximal coupling between geometry and matter represented by $f(R, T)$ theory of gravity proposed by Harko et al. [6] exploring the consequences of the coupling of matter with the geometry of the Universe instead of taking the interaction between HDE and DM (assuming that only HDE of total matter couple with geometry). In which we observed that the Hubble horizon as an $I R$ cut-off is suitable for both the models to explain the recent accelerated expansion of the Universe.
\end{abstract}

Keywords - Bianchi Type-II, VIII, IX space-time, Holographic Dark energy, $f(R, T)$ gravity.

\section{INTRODUCTION}

The HDE models have been emerged as a viable candidate to explain the problems of modern cosmology such as the recent accelerated expansion as well as the coincidence problem of the Universe in the recent years. The concept of HDE is based on the holographic principle proposed by Hooft [1] and found its roots in the quantum field theory. Cohan et al. [2] have shown that in the quantum field theory, the formation of black hole set a limit which relates cut-off to $I R$ cut-off. In the formalism of HDE, the Hubble's horizon is a most natural choice for the IR cut-off, but it leads to a wrong Equation of State of dark energy [3]. However, Pavon and Zimdhal [4], Banerjee and Pavon [5] have shown that the viable EoS of dark energy could be achieved by taking the interaction between HDE and (DM). It is strongly believed that the Universe has entered a phase of the accelerated expansion which has been confirmed by the recent observations like Supernovae-Ia, Cosmic Microwave Background Radiation, Baryon Acoustic Oscillation and Planck data. The existence of this accelerated expansion is due to of two types, one is within the framework of General Relativity (GR), the cause of the acceleration can be attributed to the existence of a mysterious component of the Universe dubbed as Dark Energy, which makes up to $70 \%$ of the total cosmic energy in the Universe and second is to modification in an action of General Relativity called Modified gravity.

Recently, a new modified gravity theory known as $f(R, T)$ gravity have proposed by Harko et al. [6], where $R$ as usual stands for the Ricci scalar and $T$ denotes the trace of energymomentum tensor. This modified theory presents a maximal coupling between geometry and matter. A number of authors have discussed the modified $f(R, T)$ gravity in different context to explain the early and late time acceleration of the universe.

The general form of the Einstein-Hilbert action for the modified $f(R, T)$ gravity in the unit $8 \pi G=1$ is as follows

$S=\frac{1}{2} \int d^{4} x \sqrt{-g}[f(R, T)+2 L m]$,

where $g$ stands for the determinant of the metric tensor $g_{i j}$, $R$ is the Ricci scalar and $T$ represents the trace of the energy-momentum tensor while $L_{m}$ denotes the matter Lagrangian density. The speed of light is taken to be unity. The energy- momentum tensor of matter is defined as

$T_{i j}=-\frac{2}{\sqrt{-g}} \frac{\delta\left(\sqrt{-g} L_{m}\right)}{\delta_{g} i j}$.

Basically this gravity is the generalization of $f(R)$ gravity based on the coupling between geometry and matter. The 
corresponding field equations have been derived in metric formalism for the various forms of $f(R, T)$.

Assuming that the Lagrangian density $L_{m}$ of matter depends only on the metric tensor components $g_{i j}$ and not on its derivatives, in this case, we obtain

$T_{i j}=g_{i j} L_{m}-\frac{\delta\left(L_{m}\right)}{\delta g}$.

Varying the action $S$ with respect to the metric tensor components $g_{i j}$, the field equation of $f(R, T)$ gravity is obtained as,

$$
\begin{gathered}
f R^{(R, T)} R_{i j}-\frac{1}{2} f(R, T) g_{i j}+f R(R, T)\left(g i j \nabla \nabla \nabla_{i}-\nabla_{i} \nabla j\right) \\
=8 \pi T_{i j}-f T(R, T) T_{i j}-f T(R, T) \Theta_{i j}
\end{gathered}
$$

where

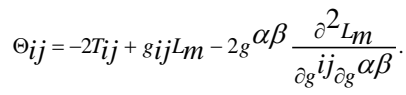

Here $f_{R}=\frac{\delta f(R, T)}{\delta R}, f T=\frac{\delta f(R, T)}{\delta T}, \Theta_{i j}={ }_{g} \alpha \beta \frac{\delta T \alpha \beta}{{ }_{\delta g} i j}$ and $\nabla_{i}$ is the covariant derivative.

The contraction of equation (4) yields

$f_{R}(R, T) R+3 \sqcap f R(R, T)-2 f(R, T)$

$$
=(8 \pi-f T(R, T)) T-f T(R, T) \Theta
$$

with $\Theta=g^{i} j_{\Theta i j}$.

The variation of stress energy of perfect fluid has the following expression

$\Theta_{i j}=-2 T_{i j}-p g_{i j}$.

On the physical nature of the matter field, the field equations also depend through the tensor $\Theta_{i j}$. Several theoretical models corresponding to different matter contributions for $f(R, T)$ gravity are possible. However, Harkoet al. (2011) gave three classes of these models

$$
f(R, T)=\left\{\begin{array}{l}
R+2 f(T) \\
f_{1}(R)+f_{2}(T) \\
f_{1}(R)+f_{2}(R) f_{3}(T)
\end{array} .\right.
$$

In this paper, we have focused on the first class $f(R, T)=R+2 f(T)$, where $f(T)$ is an arbitrary function of tress energy tensor of the form $f(T)=\mu T$ where $\mu$ is coupling constant. For this choice the gravitational field equations of $f(R, T)$ gravity become
$R_{i j}-\frac{1}{2} R g_{i j}=8 \pi T_{i j}+2 f^{\prime}(T) T_{i j}+2 f^{\prime}(T) \Theta_{i j}+f(T) g_{i j}$

where the prime denotes differentiation with respect to the argument. If the matter source is a perfect fluid then the field equations (in view of Eq. (7)) becomes

$R_{i j}-\frac{1}{2} R_{i j}=T_{i j}+\left(T_{i j}+p g_{i j}\right) f^{\prime}(T)+\frac{1}{2} f(T) g_{i j}$,

which are the field equations for non-viscous HDE of the modified $f(R, T)$ gravity theory.

In this model, let us assume the Universe filled with HDE plus pressure less Dark Matter (DM) (excluding baryonic matter), i.e.

$T_{i j}=T_{i j}^{h}+T_{i j}^{m}$,

where $T_{i j}^{h}$ and $T_{i j}^{m}$ represent the energy-momentum tensors of HDE and DM, respectively.

Many authors had described the recent accelerated expansion by assuming the interaction between HDE and DM in the different theories of gravity. In this paper, instead of taking the interaction between HDE and DM to describe the recent acceleration, we consider that the HDE interacts with the geometry of $f(R, T)$ gravity. This is due to the fact that this modified gravity theory has the interaction between matter and geometry. Therefore, we consider $T=g^{i} j_{T} h$ as the trace of energy-momentum tensor of HDE.

The Equation of State and the trace of energy-momentum tensor of HDE are respectively given by

$p h=w h \rho h$,

and

$T=\rho h-3 p h$.

Many authors have working in this area. Various forms of HDE have been discussed depending on the choices of $I R$ cut-off such as Hubble horizon, future event horizon, apparent horizon, Granda-Oliveros cut-off, etc. (the general form is $\rho h=3 c^{2} M_{p}^{2} L^{-2}$, where $c^{2}$ is a dimensionless constant, ${ }_{M} p$ stands for the reduced Planck mass and $L$ denotes the $I R$ cut-off radius). Here we consider the Hubble horizon $\left(L=H^{-1}\right)$ as an $I R$ cut-off to describe the recent acceleration.

The corresponding energy density $p h$ is given by

$p h=3 c^{2} H^{2}$.

To be more realistic, the prefect fluid Universe is just an approximation of the viscous Universe. The dissipative processes in the relativistic fluid may be modeled as bulk viscosity. The phenomenon of the bulk viscosity arises in the cosmological fluid when the fluid expands (contracts) to fast due to which the system is out of thermal equilibrium. Then, 
the effective pressure becomes negative to restore the thermal equilibrium [7]. Therefore, it is natural to consider the bulk viscosity in an accelerating Universe. It has been shown that inflation and recent acceleration can be explained using the viscous behavior of the Universe, and plays an important role in the phase transition of the Universe [8-10]. The concept of viscous DE has been discussed extensively in the literature [11]. Feng and $\mathrm{Li}$ [12] show that the age problem of the Ricci dark energy can be alleviated using the bulk viscosity. Motivated by the above works, we extend our analysis to viscous HDE with the same $I R$ cut-off which gives the recent phase transition of the universe.

The paper is organized as follows, Section I contains the introduction of related work and basics of modified $f(R, T)$ gravity, Section II contain Metric and field equations, Section III contain the solutions of the field equations to obtained non-viscous holographic dark energy cosmological models, Section IV contain the solutions of the field equations to obtained Viscous holographic dark energy cosmological models, Section V concludes summery of research work with future directions.

\section{METRIC AND FIELD EQUATIONS}

We consider a spatially homogeneous Bianchi type spacetime in the combined form as

$$
d s^{2}=d t^{2}-A^{2}\left[d \theta^{2}+f^{2}(\theta) d \varphi^{2}\right]-B^{2}[d \phi+h(\theta) d \varphi]^{2},
$$

where the scale factors $A$ and $B$ are functions of cosmic time $t$ only and $\theta, \varphi, \phi$ are the Eulerian angles. The equation (15) represents

(i) Bianchi type-II space-time if $f(\theta)=1 \quad \& \quad h(\theta)=\theta$,

(ii)Bianchi type-VIII space-time if $f(\theta)=\cosh \theta \& h(\theta)=\sinh \theta$.

(iii) Bianchi type-IX space-time if $f(\theta)=\sin \theta \& h(\theta)=\cos \theta$.

The corresponding field equations (10) for spatially homogeneous Bianchi type space-time in the combined form (15) can be written as

$$
\begin{aligned}
& 2 \frac{\dot{A}}{A} \frac{\dot{B}}{B}+\frac{\dot{A}^{2}+\delta}{A^{2}}-\frac{B^{2}}{4 A^{4}}=\rho m+\rho h \\
& \quad+(\rho m+\rho h+p h) f^{\prime}(T)+\frac{1}{2} f(T), \\
& \frac{\ddot{A}}{A}+\frac{\ddot{B}}{B}+\frac{\dot{A} \dot{B}}{A B}+\frac{B^{2}}{4 A^{4}}=-p h+\frac{1}{2} f(T), \\
& 2 \frac{\ddot{A}}{A}+\frac{\dot{A}^{2}+\delta}{A^{2}}-\frac{3 B^{2}}{4 A^{4}}=-p h+\frac{1}{2} f(T),
\end{aligned}
$$

where the overhead dot $(\cdot)$ denotes derivative with respect to the cosmic time $t$ and $\rho_{m}, \rho_{h}$ and $p_{h}$ denote the energy density of DM, the energy density of HDE and the pressure of HDE, respectively. Also, for $\delta=0,-1,+1$, the above field equations correspond to Bianchi type-II, Bianchi type-VIII and Bianchi type-IX space-time respectively.
The set of field equations (16)-(18) are only three independent equations with five unknowns $A, B, p_{h}$, $\rho_{h}$ and $\rho_{m}$. So in order to get a deterministic solution we consider the physical conditions that the shear scalar $\sigma$ is proportional to Scalar expansion $\theta$, which leads to the linear relationship between the metric potentials $A$ and $B$. i.e.

$B=A^{n}$,

where $n$ is an arbitrary constant.

From equations (17), (18) and (19), we obtain

$$
\frac{\ddot{A}}{A}+(1+n) \frac{\dot{A}^{2}}{A^{2}}+\frac{\delta}{(1-n) A^{2}}-\frac{A^{2 n-4}}{(1-n)}=0, \quad n \neq 1 .
$$

Now we discussed the solutions of the field equations to obtained cosmological Models in case of Non-viscous and viscous holographic dark energy in next sections.

\section{NON-VISCOUS HOLOGRAPHIC DARK ENERGY COSMOLOGICAL MODELS}

\section{BIANCHI TYPE-II COSMOLOGICAL MODEL:}

For $\delta=0$, the above equation (20) reduces to

$\frac{\ddot{A}}{A}+(1+n) \frac{\dot{A}^{2}}{A^{2}}-\frac{B^{2 n-4}}{(1-n)}=0, \quad n \neq 1$.

From equations (4.1), we obtain scale factor as

$$
\begin{array}{ll}
A=(\tau t+s) \frac{1}{2-n} & n \neq 2 \\
B=(\tau t+s) \frac{n}{2-n} & n \neq 2,
\end{array}
$$

where $\tau \& n$ satisfy

$2 \tau^{2} n(1-n)-(2-n)^{2}=0$,

where $\tau \neq 0 \& S$ are the arbitrary constants.

By a suitable choice of coordinates and constants [consider $\tau=1, s=0$ ], the metric (15) with the help of equations (22) and (23) can be written as

$d s^{2}=d t^{2}-(t) \frac{2}{2-n}\left[d \theta^{2}+d \varphi^{2}\right]-(t) \frac{2 n}{2-n}[d \phi+\theta d \varphi]^{2}$.

The Generalized Hubble parameter $H$ is found to be

$H=\frac{1}{3}\left(2 \frac{\dot{A}}{A}+\frac{\dot{B}}{B}\right)=\frac{(2+n)}{3(2-n) t}, \quad n \neq 2$.

The expansion scalar is found as

$\theta=3 H=\left(2 \frac{\dot{A}}{A}+\frac{\dot{B}}{B}\right)=\frac{(2+n)}{(2-n) t}, \quad n \neq 2$.

The energy density is given as

$\rho h=\frac{c^{2}(2+n)^{2}}{3(2-n) 2 t^{2}}, \quad n \neq 2$.

Anisotropic pressure of HDE is found to be 
$p h=\frac{1}{(3 \mu+2)}\left[\frac{\left(2 \mu c^{2}-27\right) n^{2}+4 n\left(2 \mu c^{2}+3\right)+4 \mu c^{2}}{6(2-n)^{2} t^{2}}\right], n \neq 2$.

Energy density of DM is found to be

$$
\rho_{m}=\frac{1}{(\mu+1)}\left[\frac{+(3 \mu+2)^{2} c^{2}(2+n)^{2}}{12(3 \mu+2)(2-n)^{2} t^{2}}\right], n \neq 2 .
$$

The Equation of State given by

$w h=\frac{1}{(3 \mu+2)}\left[\frac{\left(2 \mu c^{2}-27 n^{2}+4 n\left(2 \mu c^{2}+3\right)+4 \mu c^{2}\right.}{2 c^{2}(2+n)^{2}}\right]$.

The coincidence parameter is given by

$$
r=\left[\begin{array}{l}
\frac{4(\mu+1)(3 \mu+2) c^{2}(2+n)^{2}}{\left.12\left(12 n-n^{2}\right)(3 \mu+2)+\mu\left[2 \mu c^{2}-27\right) n^{2}+4 n\left(2 \mu c^{2}+3\right)+4 \mu c^{2}\right]} \\
+(3 \mu+2)^{2} c^{2}(2+n)^{2}
\end{array}\right] .
$$

The matter density parameter and HDE parameter are respectively given by

$\Omega_{h}=\frac{\rho_{h}}{3 H^{2}}=c^{2}$,

$\Omega m=\left[\begin{array}{l}\left.12\left(12 n-n^{2}\right)(3 \mu+2)+\mu\left[2 \mu c^{2}-27\right) n^{2}+4 n\left(2 \mu c^{2}+3\right)+4 \mu c^{2}\right] \\ \frac{+(3 \mu+2)^{2} c^{2}(2+n)^{2}}{4(\mu+1)(3 \mu+2)(2+n)}{ }^{2}\end{array}\right]$.

\section{BIANCHI TYPE-VIII COSMOLOGICAL MODEL}

For $\delta=1$, the equation (20) reduces to

$\frac{\ddot{A}}{A}+(1+n) \frac{\dot{A}^{2}}{A^{2}}-\frac{1}{(1-n) A^{2}}-\frac{A^{2 n-4}}{(1-n)}=0, \quad n \neq 1$.

We can solve the above equation and get the deterministic solution only for $n=2$.

So, from equation (35) with suitable substitution, we get

$\dot{A}^{2}=\lambda^{2}-\eta^{2} A^{2}$,

where $\lambda^{2}=-\frac{1}{3}$ and $\eta^{2}=\frac{1}{4}$

From equations (36), we get

$A=\left(\frac{\lambda}{\eta}\right) \sin (\eta t)$.

From equations (19) and (36), we get

$B=\left[\left(\frac{\lambda}{\eta}\right) \sin (\eta t)\right]^{2}$.

The metric (15) with the help of equations (37) and (38) can be written as $d s^{2}=d t^{2}-\left[\left(\frac{\lambda}{\eta}\right) \sin (\eta t)\right]^{2}\left[d \theta^{2}+\cosh ^{2} \theta d \varphi^{2}\right]-\left[\left(\frac{\lambda}{\eta}\right) \sin (\eta t)\right]^{4}$
$[d \phi+\sinh \theta d \varphi]^{2}$.

The Generalized mean Hubble parameter $H$ is found to be $H=\frac{4 \eta}{3 \tan (\eta t)}$.

The expansion scalar $\theta$ is found to be

$\theta=\frac{4 \eta}{\tan (\eta t)}$.

The energy density is given as

$\rho_{h}=\frac{16 c^{2} \eta^{2}}{3 \tan ^{2}(\eta t)}$,

Anisotropic pressure of HDE is found to be

$p h=\frac{2}{(3 \mu+2)}\left[\frac{4 \eta^{2}\left(2 \mu c^{2}-3\right)}{3 \tan ^{2}(\eta t)}+\frac{12 \eta^{2}-1}{4}\right]$.

Energy density of DM is found to be

$\rho_{m}=\frac{1}{(\mu+1)}\left[\begin{array}{c}\frac{15 \eta^{2}(3 \mu+2)-8 \eta^{2}(3 \mu+2)^{2}+4 \eta^{2} \mu\left(2 \mu c^{2}-3\right)}{3(3 \mu+2) \tan ^{2}(\eta t)}- \\ \frac{\eta^{2}}{\lambda^{2} \sin ^{2}(\eta t)}+\frac{2\left(6 \mu \eta^{2}-2 \mu-1\right)}{4(3 \mu+2)}\end{array}\right]$.

The Equation of State is given by

$w h=\frac{1}{2(3 \mu+2)}\left[\frac{4\left(2 \mu c^{2}-3\right)}{c^{2}}+\frac{3\left(12 \eta^{2}-1\right) \tan ^{2}(\eta t)}{16 c^{2} \eta^{2}}\right]$.

The coincidence parameter is given by

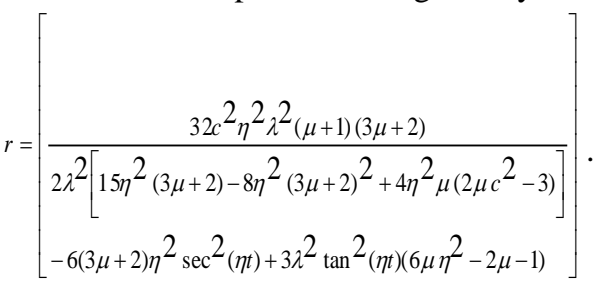

The matter density parameter and HDE parameter are respectively given by

$\Omega h=c^{2}$,

$\Omega_{m}=\frac{3 \tan ^{2}(\eta t)}{16 c^{2} \eta^{2}(\mu+1)}\left[\begin{array}{l}\frac{15 \eta^{2}(3 \mu+2)-8 \eta^{2}(3 \mu+2)^{2}+4 \eta^{2} \mu\left(2 \mu c^{2}-3\right)}{3(3 \mu+2) \tan ^{2}(\eta t)} \\ -\frac{\eta^{2}}{\lambda^{2} \sin ^{2}(\eta t)}+\frac{2\left(6 \mu \eta^{2}-2 \mu-1\right)}{4(3 \mu+2)}\end{array}\right]$.

BIANCHI TYPE-IX COSMOLOGICAL MODEL

For $\delta=1$, the equation (20) reduces to

$\frac{\ddot{A}}{A}+(1+n) \frac{\dot{A}^{2}}{A^{2}}+\frac{1}{(1-n) A^{2}}-\frac{A^{2 n-4}}{(1-n)}=0, \quad n \neq 1$. 
We can solve the above equation and get the deterministic solution only for $n=2$.So, from equation (49) with suitable substitution, we get

$\dot{A}^{2}=\lambda^{2}-\eta^{2} A^{2}$,

where $\lambda^{2}=\frac{1}{3}$ and $\eta^{2}=\frac{1}{4}$.

From equations (50), we get

$A=\left(\frac{\lambda}{\eta}\right) \sin (\eta t)$.

From equations (20) and (51), we get

$B=\left[\left(\frac{\lambda}{\eta}\right) \sin (\eta t)\right]^{2}$.

This model shows the same result as that of Bianchi typeVIII model.

The metric (15) with the help of equations (51) and (52) can be written as

$$
\begin{aligned}
& d s^{2}=d t^{2}-\left[\left(\frac{\lambda}{\eta}\right) \sin (\eta t)\right]^{2}\left[d \theta^{2}+\sin ^{2} \theta d \varphi^{2}\right]-\left[\left(\frac{\lambda}{\eta}\right) \sin (\eta t)\right]^{4} . \\
& {[d \phi+\cos \theta d \varphi]^{2}}
\end{aligned}
$$

The Generalized mean Hubble parameter $H$ is found to be

$H=\frac{4 \eta}{3 \tan (\eta t)}$.

The expansion scalar $\theta$ is found to be

$\theta=\frac{4 \eta}{\tan (\eta t)}$

The energy density is given as

$\rho h=\frac{16 c^{2} \eta^{2}}{3 \tan ^{2}(\eta t)}$.

Anisotropic pressure of HDE is found to be

$p h=\frac{2}{(3 \mu+2)}\left[\frac{4 \eta^{2}\left(2 \mu c^{2}-3\right)}{3 \tan ^{2}(\eta t)}+\frac{12 \eta^{2}-1}{4}\right]$.

Energy density of DM is found to be

$\rho_{m}=\frac{1}{(\mu+1)}\left[\begin{array}{c}\frac{15 \eta^{2}(3 \mu+2)-8 \eta^{2}(3 \mu+2)^{2}+4 \eta^{2} \mu\left(2 \mu c^{2}-3\right)}{3(3 \mu+2) \tan ^{2}(\eta t)} \\ +\frac{\eta^{2}}{\lambda^{2} \sin ^{2}(\eta t)}+\frac{2\left(6 \mu \eta^{2}-2 \mu-1\right)}{4(3 \mu+2)}\end{array}\right]$.

The Equation of State given by

$w h=\frac{1}{2(3 \mu+2)}\left[\frac{4\left(2 \mu c^{2}-3\right)}{c^{2}}+\frac{3\left(12 \eta^{2}-1\right) \tan ^{2}(\eta t)}{16 c^{2} \eta^{2}}\right]$.

The coincidence parameter is given by

$$
r=\left[\begin{array}{c}
32 c^{2} \eta^{2} \lambda^{2}(\mu+1)(3 \mu+2) \\
2 \lambda^{2}\left[15 \eta^{2}(3 \mu+2)-8 \eta^{2}(3 \mu+2)^{2}+4 \eta^{2} \mu\left(2 \mu c^{2}-3\right)\right] \\
+6(3 \mu+2) \eta^{2} \sec ^{2}(\eta t)+3 \lambda^{2} \tan ^{2}(\eta t)\left(6 \mu \eta^{2}-2 \mu-1\right)
\end{array}\right] .
$$

The matter density parameter and HDE parameter are respectively given by

$\Omega h=c^{2}$,

$$
\Omega_{m}=\frac{3 \tan ^{2}(\eta t)}{16 c^{2} \eta^{2}(\mu+1)}\left[\begin{array}{c}
\frac{15 \eta^{2}(3 \mu+2)-8 \eta^{2}(3 \mu+2)^{2}+4 \eta^{2} \mu\left(2 \mu c^{2}-3\right)}{3(3 \mu+2) \tan ^{2}(\eta t)} \\
+\frac{\eta^{2}}{\lambda^{2} \sin ^{2}(\eta t)}+\frac{2\left(6 \mu \eta^{2}-2 \mu-1\right)}{4(3 \mu+2)}
\end{array}\right]
$$

\section{VISCOUS HOLOGRAPHIC DARK ENERGY COSMOLOGICAL MODELS}

A viscous HDE with the Hubble's horizon as an $I R$ cut-off could be helpful to find the phase transition; Bulk viscosity can produce an accelerated expansion even without dark energy matter due to the presence of an effective negative pressure.

We can assume that the effective pressure of HDE is a sum of the thermo dynamical pressure $(p h)$ and the bulk viscous pressure $\pi$ i.e.

$P_{\text {eff }}=p h+\pi=p h-3 \xi H$,

where $\xi$ is the positive coefficient of the bulk viscosity.

Now, the matter Lagrangian is taken as $L_{m}=-P$ eff for which equation (20) gives $\Theta_{i j}=-2 T_{i j}-P_{\text {eff }} g_{i j}$.

To analyze the behavior of the Universe, we assume that the viscous HDE matter interacts with the geometry of the Universe. Using $f(T)=\mu T$ where $\mu$ is coupling parameter of matter with geometry. Trace $T$ of energy momentum tensor is given by

$$
T=\rho h-3(p h-3 \xi H) \text {. }
$$

From equation (64), the field equations for viscous HDE for spatially homogeneous Bianchi type metrics in the combined form in the framework of $f(R, T)$ gravity is

$$
\begin{aligned}
& 2 \frac{\dot{A}}{A} \frac{\dot{B}}{B}+\frac{\dot{A}^{2}+\delta}{A^{2}}-\frac{B^{2}}{4 A^{4}}=\rho m+\rho h \\
& \quad+(\rho m+\rho h+p h-3 \xi H) f^{\prime}(T)+\frac{1}{2} f(T), \\
& \frac{\ddot{A}}{A}+\frac{\ddot{B}}{B}+\frac{\dot{A} \dot{B}}{A B}+\frac{B^{2}}{4 A^{4}}=-p h+3 \xi H+\frac{1}{2} f(T), \\
& 2 \frac{\ddot{A}}{A}+\frac{\dot{A}^{2}+\delta}{A^{2}}-\frac{3 B^{2}}{4 A^{4}}=-p h+3 \xi H+\frac{1}{2} f(T),
\end{aligned}
$$

Equations (65)-(67) are solvable if the coefficient of bulk viscosity $\xi$ is known. Here, we assume the bulk viscous coefficient to be a constant, i.e. $\xi=\xi 0$, which is the simplest form of $\xi=\xi_{0}+\xi_{1} H$ by taking $\xi_{1}=0$. Using the solutions obtained in section III, we have obtained the various parameters for Bianchi type -II, type-VIII and type- IX viscous cosmological models as follows: 


\section{BIANCHI TYPE-II VISCOUS COSMOLOGICAL MODEL}

Anisotropic pressure of HDE is found to be

$$
p h=\frac{1}{(3 \mu+2)}\left[\begin{array}{c}
3 n(4-9 n)+2 \mu c^{2}(2+n)^{2} \\
\frac{+18 \xi 0\left(2-n^{2}\right)(3 \mu+2) t}{2 c^{2}(2+n)^{2}}
\end{array}\right], n \neq 2 .
$$

Energy density of DM is found to be

$$
\rho_{m}=\frac{1}{(\mu+1)}\left[\begin{array}{c}
3 n\left[\left(12 n-n^{2}\right)(3 \mu+2)+\mu(4-9 n)\right] \\
\frac{-8 c^{2} \mu(2+n)^{2}+12(3 \mu+2) \mu \xi 0\left(2-n^{2}\right) t}{(2-n) 2 t^{2}}
\end{array}\right], n \neq 2 .
$$

The Equation of State given by

$$
w h=\left[\frac{3 n(4-9 n)+2 \mu c^{2}(2+n)^{2}+18 \xi 0\left(2-n^{2}\right)(3 \mu+2) t}{2(3 \mu+2) c^{2}(2+n)^{2}}\right] .
$$

The coincidence parameter is given by

$$
r=\frac{(\mu+1)}{3(2-n)^{2} t^{2}}\left[\begin{array}{c}
\frac{c^{2}(2+n)^{2}}{3 n\left[\left(12 n-n^{2}\right)(3 \mu+2)+\mu(4-9 n)\right]-8 c^{2} \mu(2+n)^{2}+} \\
12(3 \mu+2) \mu(5)\left(2-n^{2}\right) t
\end{array}\right] .
$$

The matter density parameter and HDE parameter are respectively given by

$\Omega_{h}=c^{2}$,

$$
\Omega_{m}=\left[\begin{array}{l}
\left.12\left(12 n-n^{2}\right)(3 \mu+2)+\mu\left[2 \mu c^{2}-27\right) n^{2}+4 n\left(2 \mu c^{2}+3\right)+4 \mu c^{2}\right] \\
\frac{+(3 \mu+2)^{2} c^{2}(2+n)^{2}}{4(\mu+1)(3 \mu+2)(2+n)^{2}}
\end{array}\right]
$$

\section{BIANCHI TYPE-VIII VISCOUS COSMOLOGICAL MODEL}

Anisotropic pressure of HDE is found to be

$$
p_{h}=\frac{2}{(3 \mu+2)}\left[\frac{4 \eta^{2}\left(2 \mu c^{2}-3\right)}{3 \tan ^{2}(\eta t)}+\frac{12 \eta^{2}-1}{4}+\frac{2 \xi_{0} \eta(3 \mu+2)}{\tan (\eta t)}\right] .
$$

Energy density of DM is found to be

$$
\rho_{m}=\frac{1}{(\mu+1)}\left[\begin{array}{c}
\frac{15 \eta^{2}(3 \mu+2)-8 \eta^{2}(3 \mu+2)^{2}+4 \eta^{2} \mu\left(2 \mu c^{2}-3\right)}{3(3 \mu+2) \tan ^{2}(\eta t)} \\
-\frac{\eta^{2}}{\lambda^{2} \sin ^{2}(\eta t)}+\frac{2\left(6 \mu \eta^{2}-2 \mu-1\right)}{4(3 \mu+2)}+\frac{4 \xi 0 \eta \mu}{\tan (\eta t)}
\end{array}\right] .
$$

$w h=\frac{3 \tan ^{2}(\eta t)}{8 c^{2} \eta^{2}(3 \mu+2)}\left[\begin{array}{c}\frac{4 \eta^{2}\left(2 \mu c^{2}-3\right)}{3 \tan ^{2}(\eta t)}+\frac{12 \eta^{2}-1}{4} \\ +\frac{2 \xi 0 \eta(3 \mu+2)}{\tan (\eta t)}\end{array}\right]$.

The coincidence parameter is given by

$$
r=\left[\begin{array}{l}
\frac{64 \lambda^{2} c^{2} \eta^{2}(\mu+1)(3 \mu+2)}{12 \lambda^{2}\left\{15 \eta^{2}(3 \mu+2)-8 \eta^{2}(3 \mu+2)^{2}+4 \eta^{2} \mu\left(2 \mu c^{2}-3\right)\right\}-12(3 \mu+2) \eta^{2} \sec ^{2}(\eta t)} \\
+6 \lambda^{2} \tan ^{2}(\eta t)\left(6 \mu \eta^{2}-2 \mu-1\right)+12 \lambda^{2} \xi 0 \eta \mu(3 \mu+2) \tan (\eta t)
\end{array}\right] .
$$

The matter density parameter and HDE parameter are respectively given by

$\Omega_{h}=c^{2}$.

$\Omega_{m}=\frac{3 \tan ^{2}(\eta t)}{16 c^{2} \eta^{2}(\mu+1)}\left[\begin{array}{l}\frac{15 \eta^{2}(3 \mu+2)-8 \eta^{2}(3 \mu+2)^{2}+4 \eta^{2} \mu\left(2 \mu c^{2}-3\right)}{3(3 \mu+2) \tan ^{2}(\eta t)} \\ -\frac{\eta^{2}}{\lambda^{2} \sin ^{2}(\eta t)}+\frac{2\left(6 \mu \eta^{2}-2 \mu-1\right)}{4(3 \mu+2)}+\frac{4 \xi 0 \eta \mu}{\tan (\eta t)}\end{array}\right]$

\section{BIANCHI TYPE-IX VISCOUS COSMOLOGICAL MODEL}

Anisotropic pressure of HDE is found to be

$$
p h=\frac{2}{(3 \mu+2)}\left[\frac{4 \eta^{2}\left(2 \mu c^{2}-3\right)}{3 \tan ^{2}(\eta t)}+\frac{12 \eta^{2}-1}{4}+\frac{2 \xi 0 \eta(3 \mu+2)}{\tan (\eta t)}\right] \text {. }
$$

Energy density of DM is found to be

$$
\rho_{m}=\frac{1}{(\mu+1)}\left[\begin{array}{l}
\frac{15 \eta^{2}(3 \mu+2)-8 \eta^{2}(3 \mu+2)^{2}+4 \eta^{2} \mu\left(2 \mu c^{2}-3\right)}{3(3 \mu+2) \tan ^{2}(\eta t)} \\
+\frac{\eta^{2}}{\lambda^{2} \sin ^{2}(\eta t)}+\frac{2\left(6 \mu \eta^{2}-2 \mu-1\right)}{4(3 \mu+2)}+\frac{4 \xi 0 \eta \mu}{\tan (\eta t)}
\end{array}\right]
$$

The Equation of State given by

$$
w h=\frac{3 \tan ^{2}(\eta t)}{8 c^{2} \eta^{2}(3 \mu+2)}\left[\frac{4 \eta^{2}\left(2 \mu c^{2}-3\right)}{3 \tan 2(\eta t)}+\frac{12 \eta^{2}-1}{4}+\frac{2 \xi 0 \eta(3 \mu+2)}{\tan (\eta t)}\right]
$$

The coincidence parameter is given by

$$
r=\left[\begin{array}{l}
\frac{64 \lambda^{2} c^{2} \eta^{2}(\mu+1)(3 \mu+2)}{12 \lambda^{2}\left\{15 \eta^{2}(3 \mu+2)-8 \eta^{2}(3 \mu+2)^{2}+4 \eta^{2} \mu\left(2 \mu c^{2}-3\right)\right\}+12(3 \mu+2) \eta^{2} \sec ^{2}(\eta t)} \\
+6 \lambda^{2} \tan ^{2}(\eta t)\left(6 \mu \eta^{2}-2 \mu-1\right)+12 \lambda^{2} \xi 0 \eta \mu(3 \mu+2) \tan (\eta t)
\end{array}\right]
$$

The matter density parameter and HDE parameter are respectively given by

$\Omega h=c^{2}$,

$\Omega_{m}=\frac{3 \tan ^{2}(\eta t)}{16 c^{2} \eta^{2}(\mu+1)}\left[\begin{array}{l}\frac{15 \eta^{2}(3 \mu+2)-8 \eta^{2}(3 \mu+2)^{2}+4 \eta^{2} \mu\left(2 \mu c^{2}-3\right)}{3(3 \mu+2) \tan ^{2}(\eta t)} \\ +\frac{\eta^{2}}{\lambda^{2} \sin ^{2}(\eta t)}+\frac{2\left(6 \mu \eta^{2}-2 \mu-1\right)}{4(3 \mu+2)}+\frac{4 \xi 0 \eta \mu}{\tan (\eta t)}\end{array}\right]$.

The Equation of State given by 


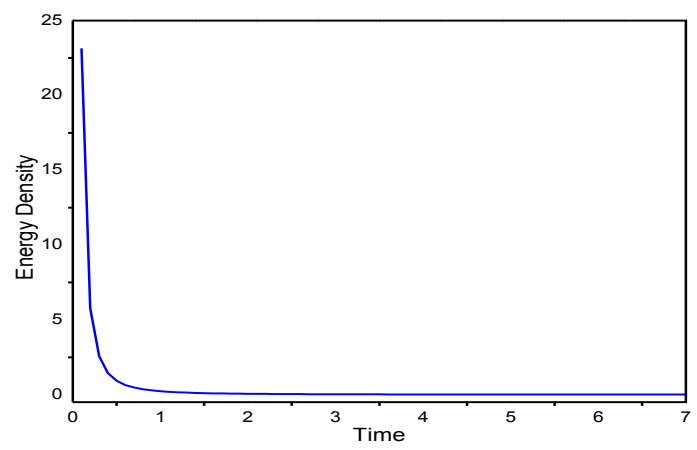

Figure (i): Energy density of the Bianchi type-II model versus cosmic time with the appropriate choice of constants.

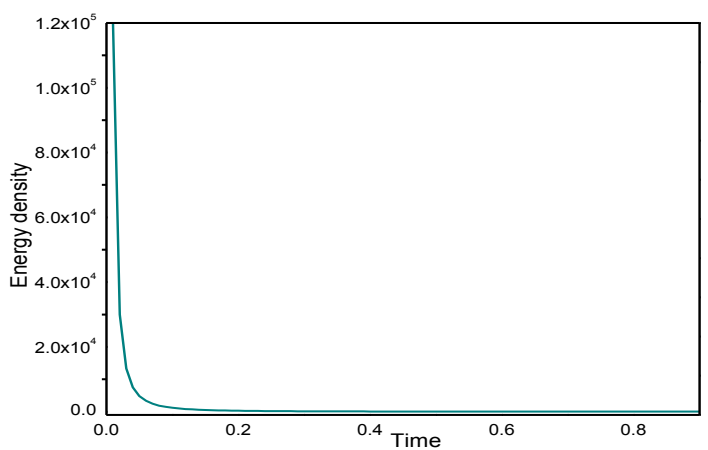

Figure (ii): Energy density of the Bianchi type-VIII model versus cosmic time with the appropriate choice of constants.

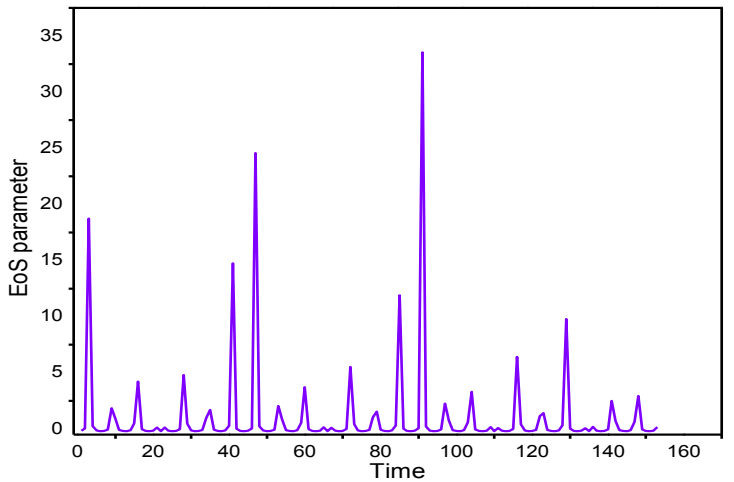

Figure (iii): Energy density of the Bianchi type-IX model versus cosmic time with the appropriate choice of constants.

\section{CONCLUSIONS}

In this paper, we have studied non-viscous and viscous HDE cosmological models with Hubble horizon as an $I R$ cut-off in the frame work of modified $f(R, T)$ gravity. The $f(R, T)$ gravity theory presents a maximal coupling between geometry and matter has been studied in this work. The consequences of the coupling of matter with the geometry of the Universe instead of taking the interaction between HDE and DM have been explored. However, we have assumed that only HDE of total matter (HDE+DM) couple with geometry. By assuming the interaction between HDE and geometry, we have investigated the possibility whether the Hubble horizon as an $I R$ cut-off could explain an accelerated expansion in $f(R, T)$ gravity. The non-viscous and viscous HDE models with Hubble's horizon as an $I R$ cut-off can explain accelerated expansion in the frame work of this modified theory.

\section{ACKNOWLEDGMENTS}

Authors are very much thankful to the anonymous referee and editor for valuable suggestions to improve this manuscript.

\section{REFERENCES}

[1 ] G. Hooft, arXiv:gr-qc/9310026

[2] A.G. Cohen, D.B. Kaplan, A.E. Nelson, Phys. Rev. Lett. 82, 4971 (1999)

[3] S.D.H. Hsu, Phys. Lett. B 594, 13 (2004)

[4] D. Pavon, W. Zimdahl, Phys. Lett. B 628, 206 (2005)

[5] N. Banerjee, D. Pav'on, Phys. Lett. B 647, 477 (2007)

[6] T. Harkoet al., Phys. Rev. D 8, 024020 (2011)

[7] A. Avelino, U. Nucamendi, J. Cosmol. Astropart. Phys. 04, 06 (2009)

[8] M.-G. Hu, X.-H. Meng, Phys. Lett. B 635, 186 (2006)

[9] C.P. Singh, S. Kumar, A. Pradhan, Class. Quantum Grav. 24, 455 (2007)

[10] P. Kumar, C.P. Singh, Astrophys. Space Sci. 357, 120 (2015)

[11] M.R. Setare, A. Sheykhi, Int. J. Mod. Phys. D 19, 1205 (2010)

[12] C-J Feng, X-Z Li, Phys. Lett. B 680, 355 (2009)

[13] S.R.Bhoyar, V.R.Chirde, Int. Jour. Scientific Research in Mathematical and Statistical Science, 5(1), 11-18, (2018).

\section{AUTHORS PROFILE}

Dr. Sanjay R Bhoyar, M.Sc (Pure Mathematics), B.Ed, M.Phil (Mathematics) Ph. D (Mathematical Sciences) from Sant Gadge Baba Amravati University,Amravati in 1993, 2014,1996, 2009\& 2013. Recently M.Sc. (Subject Communication) in 2017 from YCMOU Nashik. He is currently working as a Assistant Professor and Head Department of Mathematics (UG and PG),Phulsing Naik Mahavidyalaya, Pusad- 445216 (M.S), since 2013. He is Life member of IMS(Indian Mathematical Society).He has published 60 research papers in reputed,Peer reviewed and UGC approved Journals at national and international level which are available online. His area of research is General Relativity, Plane Gravitational Waves, Cosmology, Modified gravity theories etc. and research experience is 15 years.

Dr. Vilas R Chirde, M.Sc (Pure Mathematics), B.Ed, M.Phil (Mathematics) Ph. D (Mathematical Sciences) from Sant Gadge Baba Amravati University,Amravati $\mathrm{He}$ is currently working as an Associate Professor and Head Department of Mathematics Gopikabai Sitaramji Gawande College, Umarkhed (M.S). He is Life member of IMS (Indian Mathematical Society). He has published 65 research papers in Reputed, Peer reviewed and UGC approved Journals at national and international level which are available online. He is Ph.D. Supervisor and one scholar have awarded Ph.D. and two scholars have submitted their thesis. His area of research is General relativity, Plane Gravitational Waves, Cosmology, Modified gravity theories etc. and research experience is 20 years

Mr. Salim H Shekh, M.Sc (Pure Mathematics) from Sant Gadge Baba Amravati University,Amravati. He is currently working as a Assistant Professor, Department of Mathematics JDIET, Yavatmal. He has submitted his Ph.D thesisrecently to Sant Gadge Baba Amravati University,Amravati. .He has published 15 research papers in reputed ,Peer reviewed Journals which are available online. His area of research is Cosmology, Modified gravity theories etc. and research experience is 5 years. 